\title{
Virtual Hadronic and Leptonic Contributions to Bhabha Scattering
}

\author{
Stefano Actis, ${ }^{1}$ Michał Czakon, ${ }^{2,3}$ Janusz Gluza, ${ }^{3}$ and Tord Riemann ${ }^{4}$ \\ ${ }^{1}$ Institut für Theoretische Physik E, RWTH Aachen, D-52056 Aachen, Germany \\ ${ }^{2}$ Institut für Theoretische Physik und Astrophysik, Universität Würzburg, Am Hubland, D-97074 Würzburg, Germany \\ ${ }^{3}$ Institute of Physics, University of Silesia, Uniwersytecka 4, PL-40007 Katowice, Poland \\ ${ }^{4}$ Deutsches Elektronen-Synchrotron, DESY, Platanenallee 6, D-15738 Zeuthen, Germany
}

(Received 4 December 2007; published 2 April 2008)

\begin{abstract}
Using dispersion relations, we derive the complete virtual QED contributions to Bhabha scattering due to vacuum polarization effects. We apply our result to hadronic corrections and to heavy lepton and top quark loop insertions. We give the first complete estimate of their net numerical effects for both small and large angle scattering at typical beam energies of meson factories, the CERN Large Electron-Positron Collider, and the International Linear Collider. With a typical amount of 1-3 per mil they are of relevance for precision experiments.
\end{abstract}

The determination of the luminosity at lepton and hadron colliders is a necessary task, since in many cases the normalization of the measured cross sections is an observable of direct phenomenological interest. In practice, this task can only be solved by selecting a particular reference process, which is expected to generate large statistics, be as free as possible of systematic ambiguities and predicted by the theory to suitable accuracy. As far as lepton colliders are concerned, the above criteria are fulfilled by Bhabha scattering, i.e., the $e^{+} e^{-} \rightarrow e^{+} e^{-}$process, where a precision under the per mil level can be achieved on both the theory and the experimental sides [1-3].

In the last few years, there has been major progress in the evaluation of the corrections at the next-to-next-to-leading order accuracy. In fact, the two-loop QED corrections were first evaluated in the massless case in [4]. The photonic corrections to massive Bhabha scattering with enhancing powers of $\ln \left(s / m_{e}^{2}\right)$ were soon derived from that [5]. The missing constant term [6] plus the corrections with electron loop insertions [7,8] followed later. Recently, the heavy fermion (or $N_{f}=2$ ) corrections were derived in the limit $m_{e}^{2} \ll m^{2} \ll s,|t|,|u|[8,9]$, where $m$ is the mass of the heavy fermion, and soon after also for arbitrary $m$, with $m_{e}^{2} \ll m^{2}, s,|t|,|u|[10-12]$.

In this Letter, we present the last missing part of the virtual corrections, the hadronic ones.

The three classes of two-loop diagrams that we consider are shown in Fig. 1. They all may be evaluated by dispersion integrals, after replacing the vacuum polarization insertion $\Pi_{\text {had }}\left(q^{2}\right)$ to the photon propagator [13],

$$
\frac{g_{\mu \nu}}{q^{2}+i \delta} \rightarrow \frac{g_{\mu \alpha}}{q^{2}+i \delta}\left(q^{2} g^{\alpha \beta}-q^{\alpha} q^{\beta}\right) \Pi_{\mathrm{had}}\left(q^{2}\right) \frac{g_{\beta \nu}}{q^{2}+i \delta},
$$

by the once-subtracted dispersion integral

$$
\Pi_{\text {had }}\left(q^{2}\right)=-\frac{q^{2}}{\pi} \int_{4 M_{\pi}^{2}}^{\infty} \frac{d z}{z} \frac{\operatorname{Im} \Pi_{\mathrm{had}}(z)}{q^{2}-z+i \delta} .
$$

Finally, one relates $\operatorname{Im} \Pi_{\text {had }}$ to the hadronic cross-section ratio $R_{\text {had }}$,

$$
\operatorname{Im} \Pi_{\text {had }}(z)=-\frac{\alpha}{3} R_{\text {had }}(z)=-\frac{\alpha}{3} \frac{\sigma_{e^{+} e^{-} \rightarrow \text { hadrons }}(z)}{\left(4 \pi \alpha^{2}\right) /(3 z)},
$$

measured experimentally in the low-energy region and around hadronic resonances, and given by the perturbative QCD prediction elsewhere. For heavy fermion insertions, we have in Eq. (3) instead of $R_{\text {had }}(z)$ :

$$
R_{f}(z)=Q_{f}^{2} C_{f}\left(1+2 m_{f}^{2} / z\right) \sqrt{1-4 m_{f}^{2} / z},
$$

with electric charge $Q_{f}$ and color factor $C_{f}$, to leading order, which is sufficient for practical purposes. For leptonic or top quark intermediate states the dispersion relation approach is just an efficient technique of evaluation, but it becomes essential for light quark loops. In the case of Bhabha scattering, this method was first used some time ago for one-loop propagator insertions [14]. It was also applied to two-loop irreducible vertex (plus soft real pair) corrections [15]. Here, we derive semianalytical crosssection formulas for the so far unknown hadronic box diagrams [see Fig. 1(c)].

After replacing the photonic self-energy insertion in a two-loop diagram by the integral (2) and subsequently interchanging loop and dispersion integrations, one arrives at integrals given by the convolution of the hadronic data with kernel functions $K(z)$, where the latter represent massive one-loop Feynman diagrams. Details of the evaluation will be presented elsewhere. The one-loop self-

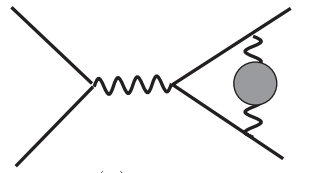

(a)

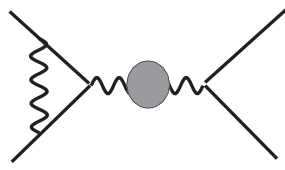

(b)

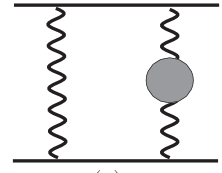

(c)
FIG. 1. Three classes of two-loop virtual hadronic Bhabha diagrams. (a) and (b) represent hadronic irreducible and reducible vertex diagrams, (c) irreducible hadronic box diagrams. 
energy kernel is trivial, $K_{\mathrm{SE}}(z)=1 /\left(q^{2}-z\right)$. Whereas the two-loop vertex kernel $K_{v}$ can be found in Eq. (5) of [15], the cross-section corrections due to the double boxes of Fig. 1(c) depend on three such kernels $K_{a}(z), a=A, B, C$ $[12,16]$. Notice that, unlike the vertex kernel, the box kernels are infrared divergent, but, analogously to the one-loop box, they have no singularity in the electron mass. The net cross-section contribution from the eight double box diagrams is still infrared divergent. These boxes become, as well as the reducible diagrams with one-loop vertices and boxes, infrared finite after adding real soft photon emission. The anatomy of that is nicely detailed in Section 2.2 of [7]. In order to construct an infrared-finite quantity, we combine: (i) Born diagrams interfering with the two-loop box diagrams [Fig. 1(c)] and with reducible vertices [Fig. 1(b)], (ii) Born diagrams with a one-loop vacuum polarization function interfering with single one-loop boxes and vertices, and finally (iii) real single-photon emission with a one-loop vacuum polarization [18]. The resulting cross section becomes

$$
\begin{aligned}
\frac{d \bar{\sigma}}{d \Omega}= & c \int_{4 M_{\pi}^{2}}^{\infty} d z \frac{R_{\text {had }}(z)}{z} \frac{1}{t-z} F_{1}(z)+c \int_{4 M_{\pi}^{2}}^{\infty} \frac{d z}{z(s-z)}\left\{R_{\text {had }}(z)\left[F_{2}(z)+F_{3}(z) \ln \left|1-\frac{z}{s}\right|\right]-R_{h}(s)\left[F_{2}(s)+F_{3}(s) \ln \left|1-\frac{z}{s}\right|\right]\right\} \\
& +c \frac{R_{h}(s)}{s}\left\{F_{2}(s) \ln \left(\frac{s}{4 M_{\pi}^{2}}-1\right)-6 \zeta_{2} F_{a}(s)+F_{3}(s)\left[2 \zeta_{2}+\frac{1}{2} \ln ^{2}\left(\frac{s}{4 M_{\pi}^{2}}-1\right)+\operatorname{Li}_{2}\left(1-\frac{s}{4 M_{\pi}^{2}}\right)\right]\right\},
\end{aligned}
$$

with $c=\alpha^{4} /\left(\pi^{2} s\right)$ and $R_{h}(s)=\theta\left(s-4 M_{\pi}^{2}\right) R_{\mathrm{had}}(s)$. Further,

$$
\begin{aligned}
& F_{1}(z)=\frac{1}{3}\left\{9 \bar{c}(s, t) \ln \left(\frac{s}{m_{e}^{2}}\right)+\left[-z^{2}\left(\frac{1}{s}+\frac{2}{t}+2 \frac{s}{t^{2}}\right)+z\left(4+4 \frac{s}{t}+2 \frac{t}{s}\right)+\frac{1}{2} \frac{t^{2}}{s}+6 \frac{s^{2}}{t}+5 s+4 t\right] \ln \left(-\frac{t}{s}\right)+s\left(-\frac{z}{t}+\frac{3}{2}\right)\right. \\
& \times \ln \left(1+\frac{t}{s}\right)+\left[\frac{1}{2} \frac{z^{2}}{s}+2 z\left(1+\frac{s}{t}\right)-\frac{11}{4} s-2 t\right] \ln ^{2}\left(-\frac{t}{s}\right)-\left[\frac{1}{2} \frac{z^{2}}{t}-z\left(1+\frac{s}{t}\right)+\frac{t^{2}}{s}+2 \frac{s^{2}}{t}+\frac{9}{2} s+\frac{15}{4} t\right] \\
& \times \ln ^{2}\left(1+\frac{t}{s}\right)+\left[\frac{z^{2}}{t}-2 z\left(1+\frac{s}{t}\right)+2 \frac{s^{2}}{t}+5 s+\frac{5}{2} t\right] \ln \left(-\frac{t}{s}\right) \ln \left(1+\frac{t}{s}\right)-4\left[\frac{t^{2}}{s}+2 \frac{s^{2}}{t}+3(s+t)\right] \\
& \times\left[1+\mathrm{Li}_{2}\left(-\frac{t}{s}\right)\right]-\left[\frac{t^{2}}{s}+2 \frac{s^{2}}{t}+3(s+t)\right] \ln \left(\frac{z}{s}\right) \ln \left(1+\frac{t}{s}\right)-\left[2 \frac{z^{2}}{t}-4 z\left(1+\frac{s}{t}\right)-4 \frac{t^{2}}{s}-2 \frac{s^{2}}{t}+s-\frac{11}{2} t\right] \zeta_{2} \\
& +\left[z^{2}\left(\frac{1}{s}+2 \frac{s}{t^{2}}+\frac{2}{t}\right)-z\left(\frac{t}{s}+2 \frac{s}{t}+2\right)\right] \ln \left(\frac{z}{s}\right)-\left[z^{2}\left(\frac{1}{s}+\frac{1}{t}\right)+2 z\left(1+\frac{s}{t}\right)+s+2 \frac{s^{2}}{t}\right] \ln \left(\frac{z}{s}\right) \ln \left(1+\frac{z}{s}\right) \\
& +\left[\frac{z^{2}}{s}+4 z\left(1+\frac{s}{t}\right)-\frac{t^{2}}{s}-4(s+t)\right] \ln \left(\frac{z}{s}\right) \ln \left(1-\frac{z}{t}\right)-\left[z^{2}\left(\frac{1}{s}+2 \frac{s}{t^{2}}+\frac{2}{t}\right)-2 z\left(\frac{t}{s}+2 \frac{s}{t}+2\right)+\frac{t^{2}}{s}+2(s+t)\right] \\
& \times \ln \left(1-\frac{z}{t}\right)+\left[\frac{z^{2}}{t}-2 z\left(1+\frac{s}{t}\right)+2 \frac{t^{2}}{s}+8 s+4 \frac{s^{2}}{t}+7 t\right] \ln \left(1-\frac{z}{t}\right) \ln \left(1+\frac{t}{s}\right)+\left[\frac{z^{2}}{s}+4 z\left(1+\frac{s}{t}\right)-\frac{t^{2}}{s}-4(s+t)\right] \\
& \times \operatorname{Li}_{2}\left(\frac{z}{t}\right)-\left[z^{2}\left(\frac{1}{s}+\frac{1}{t}\right)+2 z\left(1+\frac{s}{t}\right)+s+2 \frac{s^{2}}{t}\right] \operatorname{Li}_{2}\left(-\frac{z}{s}\right)-\left[\frac{z^{2}}{t}-2 z\left(1+\frac{s}{t}\right)+\frac{t^{2}}{s}+5 s+2 \frac{s^{2}}{t}+4 t\right] \\
& \left.\times \operatorname{Li}_{2}\left(1+\frac{z}{u}\right)\right\}+4 \bar{c}(s, t) \ln \left(\frac{2 \omega}{\sqrt{s}}\right)\left[\ln \left(\frac{s}{m_{e}^{2}}\right)+\ln \left(-\frac{t}{s}\right)-\ln \left(1+\frac{t}{s}\right)-1\right] \text {, } \\
& F_{2}(z)=\frac{1}{3}\left\{9 \bar{c}(t, s) \ln \left(\frac{s}{m_{e}^{2}}\right)-\left[z\left(\frac{t}{s}+\frac{s}{t}+2\right)-5\left(s+\frac{t}{2}+\frac{1}{2} \frac{s^{2}}{t}\right)\right] \ln \left(-\frac{t}{s}\right)-t\left(\frac{z}{s}-\frac{3}{2}\right) \ln \left(1+\frac{t}{s}\right)+\left[\frac{z^{2}}{2}\left(\frac{1}{s}+\frac{1}{t}\right)+z\left(1+\frac{t}{s}\right)\right.\right. \\
& \left.+2 \frac{t^{2}}{s}-\frac{s}{4}+\frac{3}{4} t\right] \ln ^{2}\left(-\frac{t}{s}\right)-\left[\frac{z^{2}}{2 s}-z\left(1+\frac{t}{s}\right)+2 \frac{t^{2}}{s}+\frac{s^{2}}{t}+\frac{15}{4} s+\frac{9}{2} t\right] \ln ^{2}\left(1+\frac{t}{s}\right)-\left(4 \frac{t^{2}}{s}+\frac{s^{2}}{t}+4 s+5 t\right) \ln \left(-\frac{t}{s}\right) \\
& \times \ln \left(1+\frac{t}{s}\right)-4\left[2 \frac{t^{2}}{s}+\frac{s^{2}}{t}+3(s+t)\right]\left[1+\operatorname{Li}_{2}\left(-\frac{t}{s}\right)\right]+\left(12 \frac{t^{2}}{s}+3 \frac{s^{2}}{t}+12 s+15 t\right) \zeta_{2}-\left[2 \frac{t^{2}}{s}+\frac{s^{2}}{t}+3(s+t)\right] \ln \left(\frac{z}{s}\right) \\
& \times\left[\ln \left(1+\frac{t}{s}\right)-\ln \left(-\frac{t}{s}\right)\right]+\left[z^{2}\left(\frac{1}{t}+\frac{2}{s}+2 \frac{t}{s^{2}}\right)-z\left(\frac{s}{t}+2+2 \frac{t}{s}\right)\right] \ln \left(\frac{z}{s}\right)-\left[\frac{z^{2}}{t}+4 z\left(1+\frac{t}{s}\right)-\frac{s^{2}}{t}-4(s+t)\right] \operatorname{Li}_{2}\left(1-\frac{z}{s}\right) \\
& \left.+\left[z^{2}\left(\frac{1}{s}+\frac{1}{t}\right)+2 z\left(1+\frac{t}{s}\right)+2 \frac{t^{2}}{s}+t\right] \operatorname{Li}_{2}\left(1+\frac{z}{t}\right)-\left[\frac{z^{2}}{s}-2 z\left(1+\frac{t}{s}\right)+\frac{s^{2}}{t}+2 \frac{t^{2}}{s}+4 s+5 t\right] \operatorname{Li}_{2}\left(1+\frac{z}{u}\right)\right\} \\
& +4 \bar{c}(t, s) \ln \left(\frac{2 \omega}{\sqrt{s}}\right)\left[\ln \left(\frac{s}{m_{e}^{2}}\right)+\ln \left(-\frac{t}{s}\right)-\ln \left(1+\frac{t}{s}\right)-1\right] \text {, }
\end{aligned}
$$




$$
\begin{aligned}
F_{3}(z)= & \frac{1}{3}\left\{\left[\frac{z^{2}}{s}-2 z\left(1+\frac{t}{s}\right)+4 \frac{t^{2}}{s}+2 \frac{s^{2}}{t}+7 s+8 t\right] \ln \left(1+\frac{t}{s}\right)-\left[z^{2}\left(\frac{1}{s}+\frac{1}{t}\right)+2 z\left(1+\frac{t}{s}\right)+4 \frac{t^{2}}{s}+\frac{s^{2}}{t}+3 s+4 t\right]\right. \\
& \left.\times \ln \left(-\frac{t}{s}\right)-\left[z^{2}\left(\frac{1}{t}+\frac{2}{s}+2 \frac{t}{s^{2}}\right)-2 z\left(2+\frac{s}{t}+2 \frac{t}{s}\right)+\frac{s^{2}}{t}+2(s+t)\right]\right\}, \\
F_{a}(z)= & \frac{1}{3}\left\{\left[\frac{z^{2}}{s}-2 z\left(1+\frac{t}{s}\right)+2 \frac{t^{2}}{s}+2 \frac{s^{2}}{t}+\frac{11}{2} s+5 t\right] \ln \left(1+\frac{t}{s}\right)-\left[z^{2}\left(\frac{1}{s}+\frac{1}{t}\right)+2 z\left(1+\frac{t}{s}\right)+2 \frac{t^{2}}{s}+\frac{3}{2} s+\frac{5}{2} t\right]\right. \\
& \left.\times \ln \left(-\frac{t}{s}\right)-\left[z^{2}\left(\frac{1}{t}+\frac{2}{s}+2 \frac{t}{s^{2}}\right)-2 z\left(2+\frac{s}{t}+2 \frac{t}{s}\right)-\frac{1}{2} \frac{s^{2}}{t}-s\right]\right\} .
\end{aligned}
$$

We use the abbreviation $\bar{c}(s, t)=\left[(s+t)^{2} / s+\left(2 s^{2}+\right.\right.$ $\left.\left.2 s t+t^{2}\right) / t\right] / 3$. The real soft photon emission is cut at a maximal photon energy $E_{\gamma}^{\max }=\omega$. After combining with real hard photon emission from a Monte Carlo program, the dependence on this parameter disappears. This is simulated here, as usually, by setting formally $2 \omega / \sqrt{s}=1$, which switches off the corresponding terms.
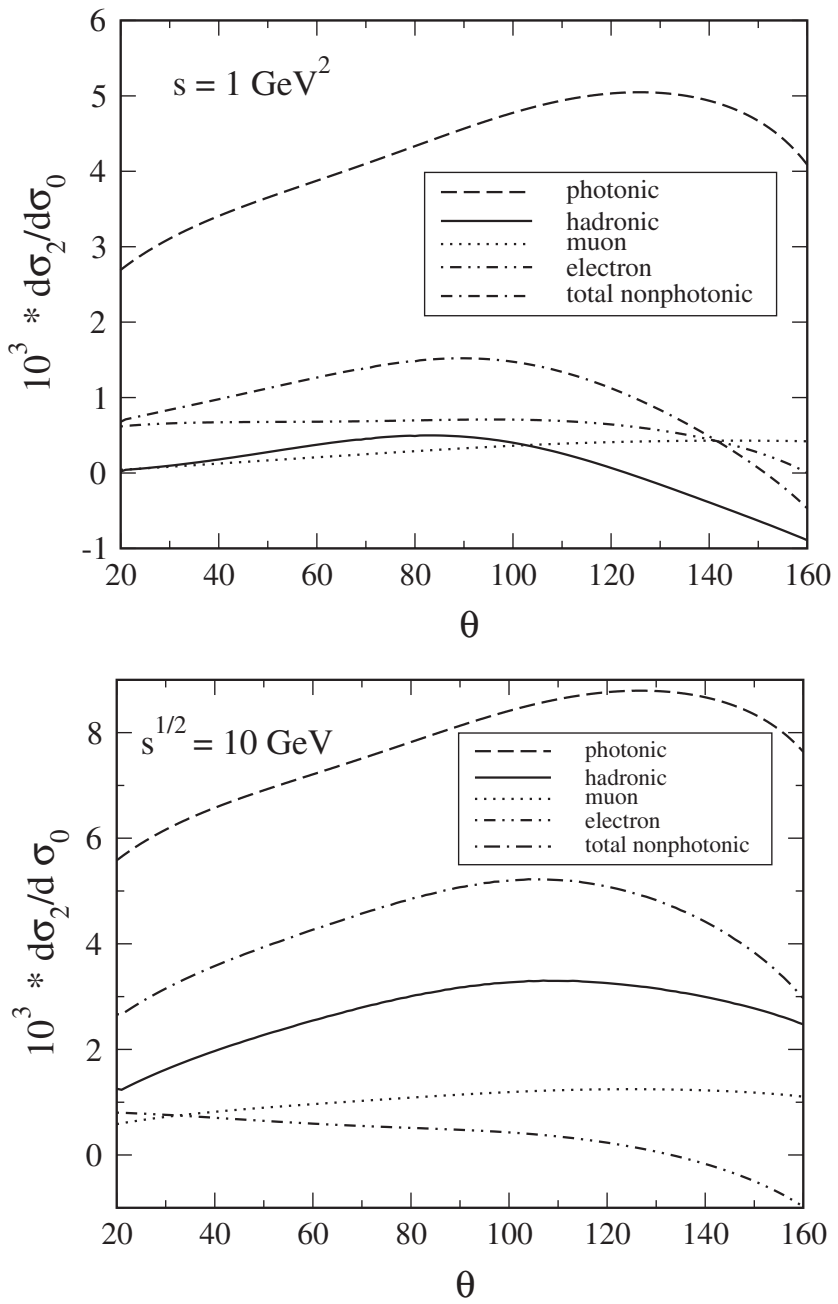

FIG. 2. Two-loop $N_{f}=2$ vertex and box corrections $d \sigma_{2}$ to Bhabha scattering in units of $10^{-3} d \sigma_{0}$ at meson factories, $\sqrt{s}=$ $1 \mathrm{GeV}$ (a) and $\sqrt{s}=10 \mathrm{GeV}$ (b).
Of course, we can get, from Eq. (5), the $N_{f}=2$ contributions from heavy fermion loop insertions, with the replacements $4 M_{\pi}^{2} \rightarrow 4 m_{f}^{2}$, and $R_{\mathrm{had}}(z) \rightarrow R_{f}(z)$; see Eq. (4) [19].

We will now discuss the numerical net effects arising from the $N_{f}=2$ vertex plus box diagrams (i.e., excluding the pure running coupling effects):
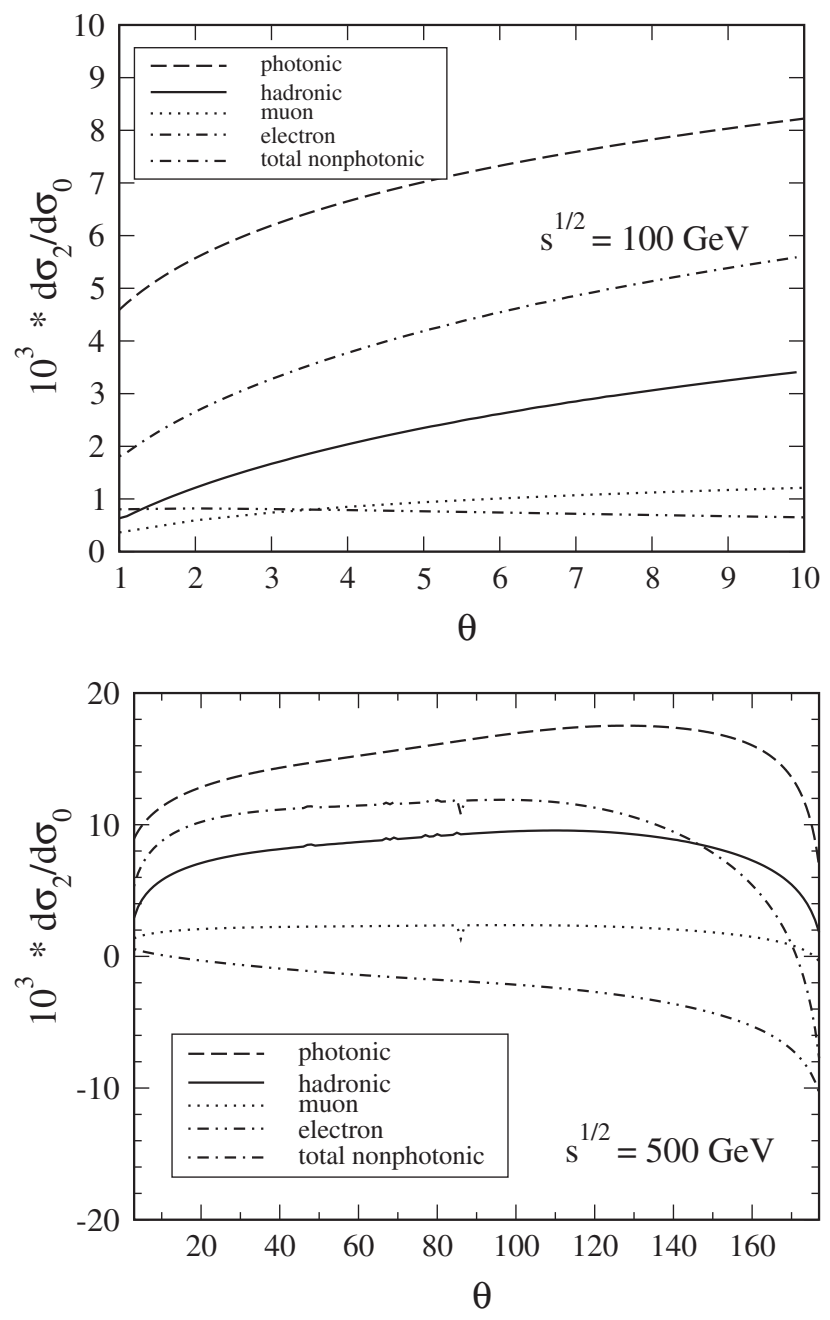

FIG. 3. Same as Fig. 2, but ILC energies of $\sqrt{s}=100 \mathrm{GeV}$ (GigaZ option) and $\sqrt{s}=500 \mathrm{GeV}$. 


$$
\frac{d \sigma_{2}}{d \Omega}=\frac{d \bar{\sigma}}{d \Omega}+\frac{d \sigma_{v}}{d \Omega}
$$

with $d \bar{\sigma} / d \Omega$ from Eq. (5). The expression for the irreducible vertex term $d \sigma_{v} / d \Omega$ derives directly from $[15,17]$. The $d \sigma_{2} / d \Omega$ is normalized to the pure photonic Bhabha Born cross section $d \sigma_{0} / d \Omega$ :

$$
\frac{d \sigma_{0}}{d \Omega}=\frac{\alpha^{2}}{s}\left(\frac{s}{t}+1+\frac{t}{s}\right)^{2}
$$

One has to compare the $d \sigma_{2} / d \Omega$ with the anticipated experimental accuracies varying from few tenths of per mil (at small angles, e.g., at LEP or ILC/GigaZ) to few per mil at large angles (at meson factories, but also at LEP and the ILC) [1-3]. It also compares to the pure photonic two-loop corrections [6], which amount to few per mil and are relevant at all energies. The large angle region at $\sqrt{s}=1 \mathrm{GeV}$ is studied, e.g., at KLOE at DAФNE [Fig. 2(a)]. Here it is also important that our formulas are valid for an arbitrary ratio $s / m_{f}^{2}$ or $s / m_{\pi}^{2}$, as long as $m_{e}^{2}$ is small. The hadronic corrections are very small, the net fermionic ones get about 1 per mil. At $\sqrt{s}=$ $10 \mathrm{GeV}$ [Fig. 2(b)], the hadronic corrections dominate and the net corrections amount to about 3 per mil. We also show the small angle region at $\sqrt{s}=100 \mathrm{GeV}$, see Fig. 3(b), which is important for the luminosity determination at LEP and the GigaZ option of the ILC. Largest are the hadronic terms, and the net effect amounts to more than 1 per mil. Finally, at $\sqrt{s}=500 \mathrm{GeV}$, the net flavor terms amount to about 8 per mil and are half of the photonic effect. In $[10,12]$, we discussed for leptons the individual terms from self-energies, irreducible vertices, and the infrared sensitive part with boxes separately. For the electron and heavy lepton corrections, there is also complete agreement with the results of a nondispersive approach [11]. As a further check on the consistency of the calculation we note that the corrections show decoupling when the masses $m_{f}^{2}$ or $M_{\pi}^{2}$ become large compared to the kinematics. In fact, the top quark and tau lepton contributions [12] decouple nearly everywhere; they are included in the total nonphotonic effects shown in the figures.

Naturally, the predictions depend on $R_{\text {had }}$, i.e., on experimental data. Although $R_{\text {had }}$ is being used in many problems (like the muon anomalous magnetic moment, for example), no recent fit is publicly available. So, we were forced to use an older version of $R_{\text {had }}$, as it was used in [15], which was available by contacting the author [20]. It is expected that current hadronic data would not induce changes larger than about $10 \%$ in our analysis. Because the size of the hadronic effects here is small enough so that only the leading digit truly matters, this is a by far sufficient accuracy.

Summarizing, the net virtual $N_{f}=2$ corrections to Bhabha scattering amount typically to $0.1 \%$. They have to be taken into account for precision studies, and a pack- age for the evaluation has been made public at [17]. For the electron and heavy lepton contributions, we agree with other computations. The newly evaluated hadronic contributions are of comparable size. These virtual corrections have to be combined yet with cut-dependent real fermion pair or hadron emissions. This has to be done with Monte Carlo generators.

We would like to thank B. Kniehl and H. Burkhardt for help concerning $R_{\text {had }}$ and A. Arbuzov, H. Czyz, S.-O. Moch, and K. Mönig for discussions. Work supported in part by GGI and INFN in Florence, by No. SFB/TRR 9 of DFG, by the Sofja Kovalevskaja Programme of the Alexander von Humboldt Foundation, and by No. MRTN-CT-2006-035505 "HEPTOOLS" and No. MRTN-CT-2006-035482 "FLAVIAnet."

[1] K. Mönig, "Bhabha scattering at the ILC", talk at Bhabha Workshop, Karlsruhe, April 2005; http://sfb-tr9.particle. uni-karlsruhe.de/.

[2] S. Jadach, "Theoretical calculations for LEP luminosity measurements", talk at Bhabha Workshop, Karlsruhe, April 2005; http://sfb-tr9.particle.uni-karlsruhe.de/.

[3] Balossini et al., Acta Phys. Polon. B 38, 3441 (2007).

[4] Z. Bern, L. Dixon, and A. Ghinculov, Phys. Rev. D 63, 053007 (2001).

[5] N. Glover, B. Tausk, and J. van der Bij, Phys. Lett. B 516, 33 (2001).

[6] A. Penin, Phys. Rev. Lett. 95, 010408 (2005).

[7] R. Bonciani, A. Ferroglia, P. Mastrolia, E. Remiddi, and J. van der Bij, Nucl. Phys. B716, 280 (2005).

[8] S. Actis, M. Czakon, J. Gluza, and T. Riemann, Nucl. Phys. B786, 26 (2007).

[9] T. Becher and K. Melnikov, J. High Energy Phys. 06 (2007) 084.

[10] S. Actis, M. Czakon, J. Gluza, and T. Riemann, XXXI Conference "Matter to the Deepest", Ustron, Poland, 5-11 Sept. 2007; http://prac.us.edu.pl/ us2007/talks.htm.

[11] R. Bonciani, A. Ferroglia, and A. Penin, arXiv:0710.4775v3.

[12] S. Actis, M. Czakon, J. Gluza, and T. Riemann, Acta Phys. Pol. B 38, 3517 (2007).

[13] N. Cabibbo and R. Gatto, Phys. Rev. 124, 1577 (1961).

[14] F. Berends and G. Komen, Phys. Lett. B 63, 432 (1976).

[15] B. Kniehl, M. Krawczyk, J. Kühn, and R. Stuart, Phys. Lett. B 209, 337 (1988).

[16] Kernels and other functions may be downloaded at [17].

[17] DESY, webpage http://www-zeuthen.desy.de/theory/ research/bhabha/.

[18] The infrared safe $N_{f}=2$ irreducible vertices [see Fig. 1(a)] and pure self-energy diagrams are not included here.

[19] In this case, the kernel integrations in Eq. (5) may be easily performed explicitly, leading to analytical expressions with Euler trilogarithms or simpler functions.

[20] H. Burkhardt, TASSO-NOTE-192, (1981); Fortran program repi.f (1986). 